\title{
CARACTERÍSTICAS ÓPTICAS E MORFOLÓGICAS DE NANOESTRUTURAS DE OURO
}

\author{
Francisco Claudece Pereira* \\ Departamento de Química, Universidade Federal do Rio Grande do Norte, CP 1524, 59072-970 Natal - RN, Brasil \\ Maria Valnice Boldrin Zanoni \\ Departamento de Química Analítica, Instituto de Química, Universidade Estadual Paulista, CP 355, 14801-970 \\ Araraquara - SP, Brasil \\ Ligia Maria Moretto e Paolo Ugo \\ Departamento de Física e Química, Universidade de Veneza, Santa Marta, 2137, 30123 Veneza - Itália
}

Recebido em 7/6/06; aceito em 14/2/07; publicado na web em 30/7/07

\begin{abstract}
OPTICAL AND MORPHOLOGICAL CHARACTERISTICS OF GOLD NANOSTRUCTURES. The present study describes a new procedure to obtain gold nanoparticles, directly in the pores of polycarbonate membranes commonly used in ultrafiltration. The dimensions of the particles may be controlled through the reduction time of the ions in the channels of the harbor matrix. The dissolution of the metallized polymer enables an investigation of the optical and morphologic properties of these elements.
\end{abstract}

Keywords: nanoparticles; colloidal gold; polycarbonate membrane.

\section{INTRODUÇÃO}

O ouro é considerado elemento químico que apresenta a maior inércia frente às agressões de natureza corrosiva. Devido a esta propriedade, o metal exibe um amplo espectro de aplicações e excelente atividade catalítica quando preparado através de técnicas específicas que possibilitem a obtenção de partículas com dimensões miniaturizadas ${ }^{1-3}$. As aplicações de ouro coloidal ou nanopartículas de ouro (NPsAu) têm se expandido recentemente graças à geração de novos dispositivos ópticos e eletrônicos, bem como possibilitado a manufatura de sondas com habilidade para detecção e/ou reconhecimento de biomoléculas de interesse da clínica médica ${ }^{4}$.

A integração entre nanotecnologia e biologia tem produzido excelentes resultados no diagnóstico molecular, na detecção de agentes químicos/biológicos e na bioengenharia. Muitos destes avanços foram alcançados graças ao desenvolvimento de sensores bioanalíticos baseados no conceito de interação em superfícies com a finalidade de capacitar um dispositivo para reações seletivas de biorreconhecimento ${ }^{5}$. Dentro deste contexto, as NPsAu apresentam algumas características particulares, tais como suas propriedades colorimétricas e condutividade, que têm potencializado seu emprego para a detecção de moléculas biológicas. A utilização de seqüências de alguns oligonucleotídeos, precursores de vários microrganismos, dentre eles, bactérias, vírus e outros agentes patogênicos na formação de conjugados com as NPsAu, tem comprovado sua alta eficiência na identificação e no diagnóstico de várias doenças, assim como no reconhecimento e detecção seqüencial de fragmentos do DNA $^{5}$. Os dispositivos criados a partir da formação destes sistemas têm atraído a atenção de distintos setores da cadeia produtiva, por exemplo, na aplicação de diagnóstico clínico, nas indústrias farmacêuticas e alimentícias, patologistas e geneticistas ${ }^{5}$.

As particularidades das NPsAu têm estimulado áreas distintas de pesquisas, nos últimos anos. A exploração das propriedades ópticas, eletrônicas e magnéticas destes materiais tem permitido seu emprego em diferentes campos de aplicação, tais como na cons-

*e-mail: claudece@ufrnet.br trução de biossensores, em sistema de liberação gradativa de drogas, lubrificantes, células solares, catálise e outros ${ }^{6}$.

Exemplos bem sucedidos têm comprovado a atividade catalítica das NPsAu para acelerar um largo número de reações, como na oxidação do $\mathrm{CO}$, redução de $\mathrm{NO}_{x}$, dissociação do $\mathrm{SO}_{2}$, na epoxidação do propeno ${ }^{3,7,8}$, redução de $\mathrm{R}-\mathrm{NO}_{2}{ }^{9}$ e na oxidação de álcoois ${ }^{10}$. Uma boa discussão sobre as propriedades catalíticas das NPsAu pode ser encontrada na literatura ${ }^{11}$.

As NPsAu apresentam a propriedade de ligar-se a macromoléculas e esta característica tem sido explorada em muitos processos de encapsulação de materiais de interesses clínico. Sun et al..$^{12}$ descreveram a utilização de NPsAu revestidas com hepatócitos e colágeno para confecção de cápsulas. A interação entre esse tipo de agente biológico e o Au ocorre através da mimetização de ligação cruzada e destaca a eficiência mecânica do material encapsulado. Similarmente, Wang e colaboradores ${ }^{13,14}$ desenvolveram uma estratégia de imobilização de materiais biológicos em NPsAu de $15 \mathrm{~nm}$. O procedimento consiste na montagem de filmes poliméricos dotados de terminais amina, capazes de incorporar sistemas específicos, tais como a proteína "A" e o anticorpo haptoglobina. $\mathrm{O}$ dispositivo permite sua aplicação em imunoensaios e pode ser usado como modelo de avaliação de sistemas mais complexos. Krpetic e colaboradores ${ }^{15}$ empregaram conjugados de NPsAu com habilidade para reconhecimento especifico de células cancerosas do tipo K562 in vivo. O sistema foi utilizado no mapeamento de células defeituosas em ratos e tratamento através de irradiação com raios-X. A alta sinergia entre as partículas de Au e o tratamento radioativo in situ permitiu a redução e/ou erradicação de tumores.

A propriedade das NPsAu na formação de conjugados tem sido explorada para gerar sistemas carreadores. Alguns pesquisadores ${ }^{4,16}$ têm mostrado a exeqüibilidade na preparação de partículas de $\mathrm{Au}$ com derivados de tetratiofulvalenos e de polimetilmetacrilato. As NPsAu são inseridas em uma solução do substrato a fim de gerar o conjugado desejado e o sistema apresenta potencialidades para aplicação na engenharia de polímeros com propriedades funcionalizadas. Zhang et al. ${ }^{17}$ mostraram a viabilidade na síntese de um derivado fulerênico $\left(\mathrm{C}_{60}\right)$ de natureza anfifílica, a partir de sua agregação em partículas de ouro de $10 \mathrm{~nm}$. O procedimento 
permite a geração de esferas miniaturizadas e a possibilidade de sua aplicação em meio aquoso e orgânico.

Uma das áreas da Química que mais tem explorado as propriedades das NPsAu é a eletroanálise. Diversos experimentos comprovam a eficiência dessas partículas na construção de sensores ou na modificação de superfícies eletródicas para fins de imunoensaios. Dentre os mais relevantes, destacam-se: Wang e colaboradores ${ }^{18}$ empregaram ditiotreitol e dodecanotiol na formação de filmes automontados em superfícies eletródicas e a subseqüente incorporação de NPsAu através da afinidade dos grupos sulfurados com o metal. O dispositivo foi aplicado para determinação de epinefrina em valores de concentração de $6,0 \times 10^{-8} \mathrm{~mol} \mathrm{~L}^{-1}$. Similarmente, Zhao et al. ${ }^{19}$ mostram ser possível gerar NPsAu diretamente na superfície de um eletrodo de Au maciço através da aplicação de potencial de $10 \mathrm{~V}$. Os óxidos formados nesta etapa são reduzidos a $\mathrm{Au}^{0}$ através de um agente redutor. $\mathrm{O}$ dispositivo foi aplicado para quantificação de carboidratos.

As NPsAu também podem ser utilizadas na metalização de filmes automontados. Diferentes experimentos mostraram ser possível a modificação de superfície de eletrodos para a análise de $\alpha-1$ fetoproteína ${ }^{20}$, dopamina na presença de ácido ascórbico ${ }^{21}$, o anticorpo encefalite $B$ japonê s $^{22,23}$, os antígenos Schistosoma japonicum $^{24}$ e hepatite $B^{25}$ além da bactéria difteria ${ }^{26}$.

De acordo com a literatura, poucos procedimentos têm sido primordiais para a geração de NPsAu. Dentre os mais relevantes destacam-se aqueles que utilizam a redução do $\mathrm{HAuCl}_{4}$ com boridreto $^{6,15,21-24,27-29}$ ou silicatos ${ }^{10,30,31}$. Outras metodologias mostraram a exeqüibilidade na utilização de radiação a laser ${ }^{32}$ ou processos sono-químicos ${ }^{33}$ a partir de uma superfície do metal; a redução eletroquímica de íons de $\mathrm{Au}$, diretamente nos poros de matrizes à base de filmes de alumina anodizada, também tem sido utilizada ${ }^{34}$.

$\mathrm{O}$ uso de membranas de policarbonato (MPCs), comumente empregadas em processos de ultrafiltração, apresenta considerável destaque nos últimos anos como modelo de síntese de nanoestruturas de ouro. Estas MPCs caracterizam-se por apresentar poros cilíndricos em toda a sua extensão e viabilizam a geração de fios de $\mathrm{Au}$ para os propósitos mais diversificados. O procedimento para a deposição química do metal nos poros de uma MPC é bem documentado na literatura ${ }^{35-38}$.

Este trabalho teve como objetivos descrever o comportamento óptico e morfológico de estruturas de ouro coloidal, obtidas após a redução e subseqüente deposição de íons de ouro diretamente nos poros de MPCs e o efeito da dissolução da matriz hospedeira para a liberação destes elementos.

\section{PARTE EXPERIMENTAL}

Todas as medidas espectrofotométricas foram realizadas em um espectrofotômetro Lamda 2 da Perkin Elmer, utilizando-se cubetas de quartzo de $1 \mathrm{~cm}$ de caminho óptico. Os estudos morfológicos das membranas douradas e dos nanoelementos foram realizados empregando-se técnicas de microscopia eletrônica de varredura de superfície (MEV) com instrumento JEOL JSM 5600 e microscopia eletrônica de transmissão (MET) JEOL JEM 3010, ambos da Philips e equipados com um espectroscópio de dispersão de energia (SDE). As fotos dos pedaços de membranas foram obtidas através de máquina digital Cyber-shot da Sony com resolução de 4,1 Mpixels acoplada a um microscópio óptico binocular convencional.

Os reagentes utilizados foram ácido trifluoracético, nitrato de prata, ácido nítrico, diclorometano, cloreto de estanho II, solução amoniacal, metanol e sulfito de sódio. Formaldeido foi empregado como catalisador no processo redox dos íons de Au. Todos os reagentes empregados apresentaram grau analítico. A solução de douração Oromerse ${ }^{\circledR}$ foi fornecida pela Italgalvano SPA - Itália e as MPCs foram fornecidas pela Osmonics Laboratory Products USA com poros de 10, 30 e $220 \mathrm{~nm}$, diâmetro de $47 \mathrm{~mm}$ e $6 \mu \mathrm{m}$ de espessura.

\section{RESULTADOS E DISCUSSÃO}

\section{Caracterização óptica}

O estudo óptico dos compósitos MPC/NPsAu, obtidos durante o processo de preenchimento lento e gradual dos poros da membrana com ouro elementar, foi realizado por meio da deposição do metal, conforme especificação ${ }^{35}$. A análise das propriedades ópticas destes materiais foi conduzida avaliando-se o efeito do tempo de deposição de ouro tomando, inicialmente, uma MPC com poros de $30 \mathrm{~nm}$, como única matriz polimérica para a recepção dos elementos. Esse ensaio foi efetuado através da imersão da membrana pré-ativada em solução de sulfito áurico de sódio, na presença do catalisador formaldeído a uma temperatura controlada a $\mathrm{O}^{\circ} \mathrm{C}$, utilizando-se um criostato por um período máximo de até $24 \mathrm{~h}$. Após o início da etapa de deposição química do metal na MPC, a matriz foi removida da solução de douração, em tempos devidamente programados, e efetuou-se um corte longitudinal de aproximadamente $6,7 \mathrm{~mm}$ de largura. Após a remoção de cada pedaço da membrana, prosseguiu-se com o processo de redução dos íons de ouro na MPC remanescente até atingir as etapas desejadas. Os tempos de douração da MPC investigados foram 0,$5 ; 0,8 ; 1,0 ; 1,3 ; 1,7 ; 2,0$ e 24 h. Concluída esta operação, os pedaços da membrana foram imersos, em separado, em solução de ácido nítrico $50 \%$ (v/v) para a remoção de resíduos dos reagentes empregados em todo o processo. A seguir, estes pedaços da MPC dourada foram submetidos à análise em microscópio óptico e registradas as suas fotos correspondentes. Os resultados deste estudo são mostrados na Figura 1.

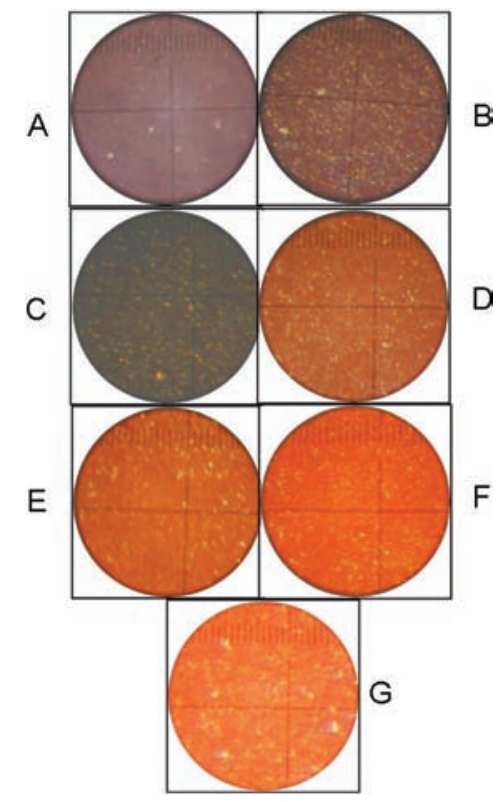

Figura 1. Fotomicrografias de uma única MPC com poros de $30 \mathrm{~nm}$ submetida à deposição química de ouro em função do tempo de douração. Figuras: $A=$ 0,$5 ; B=0,8 ; C=1,0 ; D=1,3 ; E=1,7 ; F=2,0$ e $G=24$ de deposição de $\mathrm{Au}$, respectivamente

A análise da Figura 1 mostra a geração de diferentes tonalidades, para cada pedaço da MPC, que oscilam em função do tempo de exposição da matriz à solução de douração. Os resultados evi- 
denciam um processo de crescimento e deposição, lento e gradual, das partículas de ouro nos canais da MPC em função do aumento do tempo reacional, proporcionando a formação de estruturas maiores e teores mais elevados de ouro elementar na MPC. Estes resultados estão em concordância com a literatura ${ }^{36,39}$, que relata a obtenção de diferentes colorações observadas para a redução de íons ouro sobre um conjunto de membranas de alumina. Contudo, os trabalhos prévios ${ }^{36,39}$ foram realizados através da fixação de um único tempo de douração para várias matrizes de alumina, cada uma com diferentes porosidades. Em outras palavras, usou-se um total de 25 membranas de alumina com diâmetros na ordem de 20 a $150 \mathrm{~nm}$, para inferir o efeito do crescimento das estruturas de ouro em função da magnitude dos canais de cada matriz utilizada para recepção do metal nobre.

De acordo com esses relatos ${ }^{36,39}$, as diversas tonalidades fornecidas pelos arranjos de ouro depositados em uma matriz são, fundamentalmente, dependentes dos diâmetros e comprimentos desses elementos. Desta forma, mudando a dimensão dos poros das matrizes utilizadas, é possível obter partículas/fios com magnitudes correspondentes aos respectivos canais das membranas hospedeiras. Todavia, o diâmetro das partículas está limitado ao diâmetro dos poros da matriz, enquanto o comprimento dos fios de $\mathrm{Au}$, que se formam em tempos maiores da redução dos íons ouro, relaciona-se com a espessura da matriz empregada, podendo alcançar a escala micrométrica. Assim, as propriedades ópticas destes materiais estão intrinsecamente relacionadas à razão de aspecto, que envolve o comprimento e o diâmetro de tais estruturas.

O comportamento observado na Figura 1 permite depreender que as distintas colorações obtidas são resultantes de geometrias e formas em crescimento, que se desenvolvem em função do aumento no tempo de deposição de ouro na matriz polimérica. Não obstante, os resultados obtidos com a utilização de somente uma MPC de $30 \mathrm{~nm}$ mostra que é possível monitorar e interromper o crescimento destes elementos, controlando, assim, o tamanho das partículas desejadas, simplesmente através de um rigoroso controle no tempo de deposição do metal. Esses resultados comprovam que o emprego de várias membranas de diferentes características não é determinante para a geração de estruturas distintas para fins específicos.

Nanomateriais apresentam interessantes propriedades ópticas. Um exemplo clássico desse comportamento são as partículas em suspensão coloidal de ouro, as quais podem exibir diversas tonalidades na região do visível. Esta particularidade é intrinsecamente dependente da forma e das dimensões destes constituintes ${ }^{34}$. A diversidade de cores observada para estes materiais é resultante da ressonância plasmática de superfície (RPS) destas partículas e tem sido devidamente explicada através da teoria de MaxwellGarnett ${ }^{40,41}$. Essa propriedade fornece uma possibilidade de investigação sobre o comportamento espectrofotométrico das partículas de ouro coloidal na região visível do espectro eletromagnético. Considerando que as próprias MPCs são opticamente transparentes na região de interesse $\mathrm{e}^{36,39}$ e que apresentam pronta solubilidade em vários solventes orgânicos, a dissolvência (em separado) dos pedaços da MPC dourada em diclorometano preserva as propriedades ópticas dos elementos anteriormente hospedados em seus canais e as suspensões adquirem colorações diferenciadas, comprovando que é possível se obter partículas com diferentes características em uma única matriz, sendo esta relação função somente do tempo de crescimento destas estruturas.

As diferentes cores observadas nas suspensões do ouro coloidal são devido às oscilações coletivas dos elétrons responsáveis pelas bandas de condução existentes na superfície das NPsAu. Essas entidades interagem com o vetor elétrico da energia radiante, consti- tuindo um fenômeno denominado de ressonância de polaritons superficiais, sendo resultante de vibrações eletrônicas e mudanças na densidade de carga localizada em uma interface, também conhecido como ondas de Langmuir. De acordo com a literatura ${ }^{28,34,42}$, as NPs metálicas comportam-se eletronicamente como caixas quânticas ou "quantum dots" e são governadas pelas regras da mecânica quântica. A mobilidade dos elétrons livres promove a oscilação da frequiência da RPS, cuja excitação pode ser observada na região do visível em partículas miniaturizadas. Materiais nanoparticulados são dotados de uma lacuna envolvendo as bandas de valência e de condução, capazes de permitir uma transição eletrônica na interface condutor-isolador. Partículas metálicas com magnitudes ao redor de $20 \mathrm{~nm}$ são susceptíveis aos efeitos de quantização e este fenômeno se manifesta como uma função dependente de suas dimensões, justificando, assim, a observação de tonalidades distintas para as suspensões de ouro coloidal ${ }^{43}$.

O comportamento espectrofotométrico destas estruturas é bem descrito na literatura ${ }^{37,38,40,41,44}$. Todavia, esses estudos relatam o emprego da radiação eletromagnética incidindo diretamente sobre os próprios pedaços das membranas com os seus respectivos hóspedes (análise no estado sólido). Considerando que as MPCs são prontamente solúveis em diclorometano e que sua dissolução é capaz de promover a liberação dos elementos agregados em seus canais, possibilitando uma melhor investigação desses materiais em um meio totalmente homogêneo, efetuou-se uma análise sobre as características ópticas das suspensões de ouro coloidal obtidas após a dissolvência da matriz previamente metalizada. Os resultados são mostrados na Figura 2.

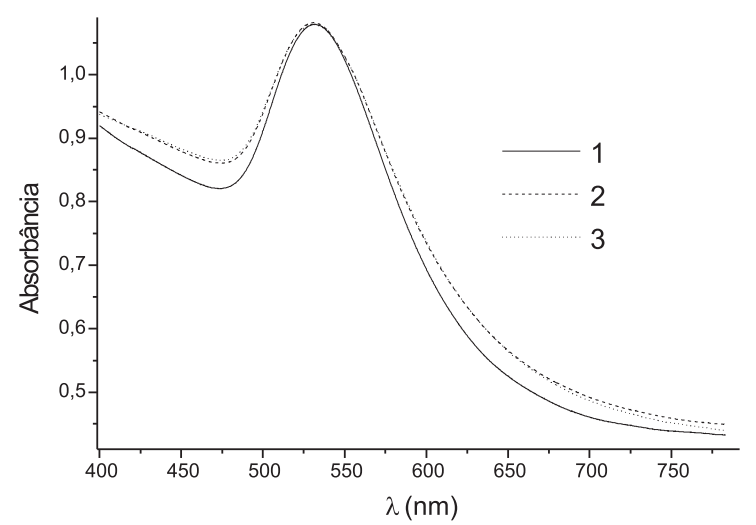

Figura 2. Espectros de RPS obtidos dos pedaços de uma MPC com poros de $30 \mathrm{~nm}$, previamente metalizada com posterior dissolução em diclorometano. Curva $1=0,5 ;$ curva $2=0,8$ e curva $3=1,0$ h de douração

Os espectros caracterizam-se por apresentar um pico único e bem definido de RPS centrado em $\lambda_{\text {max }}=532 \mathrm{~nm}$. Estes resultados são coincidentes com aqueles observados para elementos similares retidos em membranas hospedeiras ${ }^{37,38,40,41,44}$. Embora as suspensões apresentem colorações diferenciadas, em função do tempo de exposição à solução de douração, nenhum deslocamento no $\lambda$ de RPS foi observado.

A frequiência de RPS em uma partícula manifesta-se como uma função de vários parâmetros, dentre eles a natureza do elemento e seu ambiente, sua escala, geometria, sua constante dielétrica, dentre outros $^{28,34}$. Assim, a teoria de Maxwell-Garnett ${ }^{40,41}$ prevê que partículas metálicas, cujos raios são infinitesimalmente menores em relação ao comprimento de onda da luz visível, devem apresentar características ópticas semelhantes. Mudanças neste comportamento somente devem ser observadas quando a razão de aspecto (relação entre comprimento e diâmetro) desses elementos assume dimensões 
maiores ${ }^{34}$. Dentro deste contexto, os resultados espectrofotométricos mostram que, no intervalo de tempo investigado $(0,5 ; 0,8$ e $1,0 \mathrm{~h}) \mathrm{de}$ redução dos íons de Au nos poros da MPC de $30 \mathrm{~nm}$, as partículas geradas assumem magnitudes nanométricas.

De acordo com a literatura ${ }^{37,38,40,44}$, partículas obtidas em matrizes com diâmetros diferenciados, além de mostrarem características diversas, também apresentam comportamento óptico distinto. Nesta conjuntura, avaliou-se o efeito da porosidade de várias MPCs com diâmetros de 10, 30 e $220 \mathrm{~nm}$, submetidas a iguais processos de douração seguido de sua dissolução em diclorometano, para liberação dos fios de ouro. Para este ensaio, foi fixado um tempo de $24 \mathrm{~h}$ de deposição de ouro para cada uma das membranas utilizadas. Nestas condições, os canais das MPCs são completamente preenchidos de ouro elementar, sendo a magnitude destas estruturas limitada à espessura e ao diâmetro dos poros das próprias MPCs. Assim, é possível a obtenção de fios de ouro, cujos comprimentos podem alcançar $6 \mu \mathrm{m}$ e diâmetros correspondentes àqueles das matrizes, resultando em um considerável aumento na razão de aspecto para estes elementos. Os resultados espectrofotométricos obtidos nesta investigação são mostrados na Figura 3.

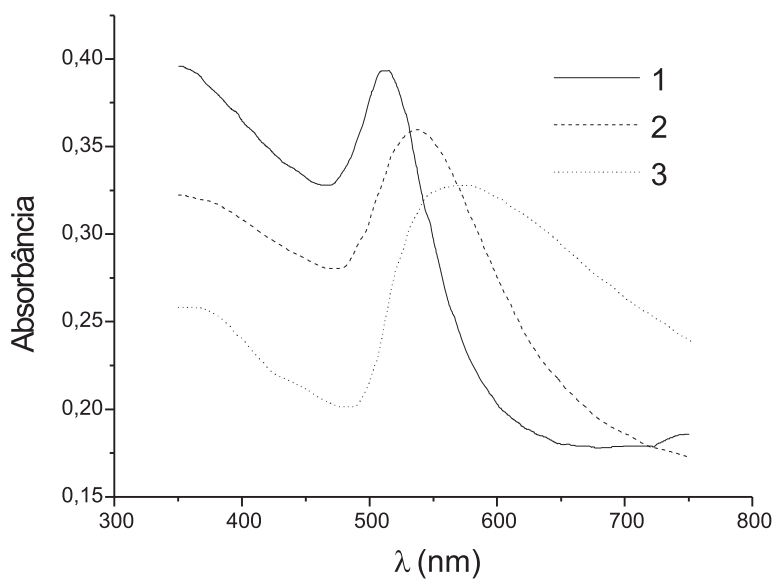

Figura 3. Espectros de RPS obtidos de pedaços das MPCs douradas por um período de 24 h e posterior dissolução em diclorometano. Curva $1=M P C$ com poros de $10 \mathrm{~nm}$; curva $2=$ MPC com poros de $30 \mathrm{~nm}$ e curva $3=\mathrm{MPC}$ com poros de $220 \mathrm{~nm}$

Embora a literatura $37,38,40,41,44$ relate que o comportamento espectrofotométrico dos cilindros de ouro agregados nas MPCs (análise no estado sólido) apresenta deslocamento nos $\lambda$ de RPS e diminuição na intensidade do sinal, em função do aumento na dimensão dos poros da matriz, o mesmo efeito pode ser constatado em suspensões coloidais de ouro obtidas após a dissolvência das MPCs previamente douradas. Este fenômeno é devido ao aumento na razão de aspecto para estes elementos e devidamente explicado pela teoria de Maxwell-Garnett ${ }^{40,41}$. Efeito similar também tem sido relatado no emprego de NPsAu utilizadas como suporte para ancorar materiais biológicos ${ }^{5}$. De acordo com os estudos pioneiros desenvolvidos por $\mathrm{Mie}^{5}$, os espectros de RPS têm origem na interação dos elétrons $6 \mathrm{~s}$ destas partículas com a energia radiante, sendo resultante do somatório das contribuições de absorção e espalhamento da luz. Para partículas com dimensões menores que $25 \mathrm{~nm}$, nenhum deslocamento nos $\lambda$ pode ser observado; contudo, para magnitudes maiores, é possível constatar deslocamento para o vermelho e atenuação na intensidade do sinal ${ }^{5}$.

Uma vez que os resultados prévios permitem depreender que é possível monitorar e controlar o crescimento destas estruturas no interior de uma única MPC, e a propriedade desta em se dissolver em alguns solventes orgânicos com liberação de seus constituintes, e considerando ainda que o emprego de técnicas de microscopia eletrônica de transmissão (MET) pode fornecer imagens com alta resolução, que possibilitam uma avaliação morfológica das etapas de crescimento destes arranjos, promoveu-se um novo processo de douração de uma única MPC com poros de $30 \mathrm{~nm}$, utilizando-se o processo padrão e a realização de cortes longitudinais da matriz em função do tempo de deposição do ouro. Este novo estudo foi conduzido através da tomada de dois tempos, de 0,2 e $5 \mathrm{~h}$, de douração da MPC seguidos de sua dissolução. As características estruturais dos elementos obtidos após a dissolvência da matriz podem ser visualizadas na Figura 4.

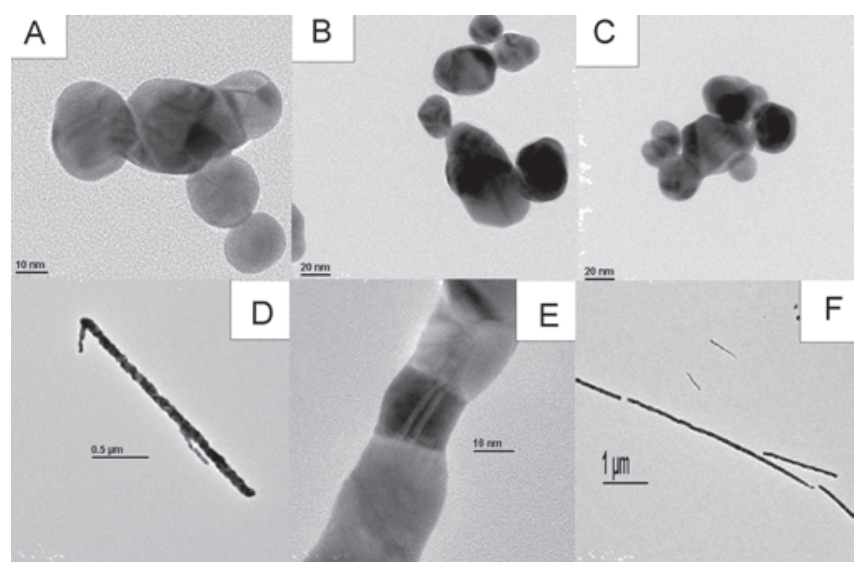

Figura 4. Imagens MET das estruturas de ouro obtidas após a dissolução em diclorometano de pedaços de uma MPC com poros de $30 \mathrm{~nm}$, dourada por um período de: $(A, B$ e $C)=0,2$ he $(D, E$ e $F)=5 h$

A análise das referidas imagens mostra a fase inicial de formação das NPsAu (Figura 4 A-C) após 0,2 h e a fase final (Figura 4 DF) após 5 h de deposição de ouro. A comparação destes resultados confirma que é possível intervir no processo de crescimento das partículas, controlando, rigorosamente, o tempo de sua deposição. Desta forma, dependendo do trabalho que se deseje realizar com estes materiais, o controle no tempo de evolução das partículas permite a obtenção de geometrias e magnitudes diferenciadas entre si. Em adição, os resultados possibilitam depreender que a deposição de ouro, ocorrida em até $5 \mathrm{~h}$, é o tempo suficiente para a estruturação das fibras no interior dos canais da MPC. Em tempos superiores de redução dos íons de ouro, a deposição passa a ocorrer na superfície da matriz para formação dos extratos do metal nas duas faces da $\mathrm{MPC}^{35}$.

A aplicação de técnicas de microscopia eletrônica de varredura (MEV) permite investigar as características morfológicas e topográficas de materiais na escala miniaturizada ${ }^{38}$. Neste sentido, as estruturas de ouro, obtidas no processo final de douração, foram avaliadas para a extração de seus parâmetros dimensionais, tais como diâmetro e comprimento. A Figura 5 mostra as possíveis magnitudes destes fios obtidos após dissolução de uma MPC com poros de $30 \mathrm{~nm}$, previamente dourada por um período de $24 \mathrm{~h}$.

A Figura 5A mostra os filamentos de ouro íntegros em toda a sua extensão obtidos após a completa douração da matriz, seguido de sua dissolução em diclorometano. Na Figura 5B, é possível inferir as dimensões centrais destes fios, os quais apresentam diâmetro médio de $87 \pm 5 \mathrm{~nm}$. Contudo, nas extremidades destes bastões, este valor é acentuadamente menor. A largura central de $87 \mathrm{~nm}$ excede os $30 \mathrm{~nm}$ dos poros das MPCs utilizadas, conforme especificação do fabricante e outros pesquisadores ${ }^{37}$. As razões que justificam este 


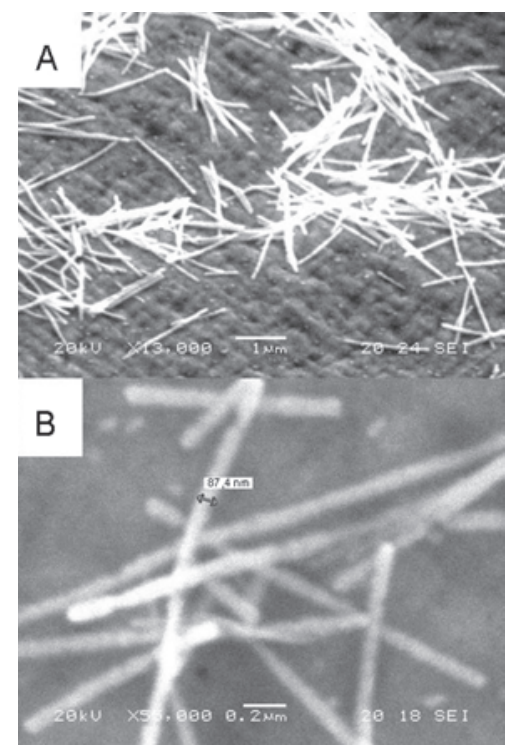

Figura 5. Imagens $M E V$ de uma MPC com poros de $30 \mathrm{~nm}$, dourada por um período de 24 h e posterior dissolução em diclorometano

fenômeno são relatadas por Schonenberger ${ }^{45}$ que observou um crescimento na ordem de 2,5 vezes entre as extremidades e o centro destes filamentos e um aumento de cerca de 6 vezes na área dos poros das matrizes após seus preenchimentos. De acordo com trabalhos $\operatorname{prévios}^{45}$, as membranas não apresentam uniformidade no diâmetro de seus poros e a pressão interna, exercida pelos filamentos durante seu processo de evolução, mostra uma tendência natural no sentido de distender o diâmetro original da matriz.

\section{CONCLUSÃO}

O presente estudo descreve as fases de crescimento e agregação de nanopartículas de ouro geradas por meio da redução química dos íons a $\mathrm{Au}$ elementar e o subseqüente desenvolvimento dos filamentos nos poros das matrizes poliméricas. Os resultados evidenciam que a estruturação destes elementos pode ser condicionada, a partir de um rigoroso controle no tempo de deposição do metal nos canais de uma única MPC. Assim, é possível a obtenção de formas e geometrias distintas para estas partículas, bem como a construção de bastões com dimensões desejadas para sua aplicação em sistemas específicos. A dissolução da matriz em diclorometano promove a liberação destas estruturas, com formação de uma suspensão coloidal estável e homogênea em toda a sua extensão, permitindo a exploração de aplicações diversificadas para estes materiais.

\section{AGRADECIMENTOS}

À CAPES e à FAPESP pelo suporte financeiro para a realização deste trabalho e aos(as) assessores(as) da Revista Química Nova pelas valiosas sugestões.

\section{REFERÊNCIAS}

1. Ma, X.; Li, X.; Lun, N.; Wen, S.; Mater. Chem. Phys. 2006, 97, 351.

2. Chen, J. H.; Lin, J. N.; Kang, Y. M.; Yu, W. Y.; Kuo, C. N.; Wan, B. Z.; Appl. Catal., A 2005, 291, 162.
3. Akolekar, D. B.; Bhargava, S. K.; J. Mol. Catal. A: Chem. 2005, 236, 77.

4. Guo, W. J.; Dai, J.; Zhang, D. Q.; Bian, Q. Y. Z. G. Q.; Inorg. Chem. Commun. 2005, 8, 994.

5. Kim, C. K.; Kalluru, R. R.; Singh, J. P.; Fortner, A.; Griffin, J.; Darbha, G. K.; Ray, P. C.; Nanotechnology 2006, 17, 3085.

6. Page, K.; Proffen, T.; Terrones, H.; Terrones, M.; Lee, L.; Yang, Y.; Stemmer, S.; Seshadri, R.; Cheetham, A. K.; Chem. Phys. Lett. 2004, 393, 385.

7. Molina, L. M.; Hammer, B.; Appl. Catal., A 2005, 291, 21.

8. Bandyopadhyay, M.; Korsak, O.; Berg, M. W. E.; Grunert, W.; Birkner, A.; Li, W.; Schuth, F.; Gies, H.; Microporous Mesoporous Mater. 2006, 89, 158

9. Chen, Y.; Qiu, J.; Wang, X.; Xiu, J.; J. Catal. 2006, 242, 227.

10. Mertens, P. G. N.; Bulut, M.; Gevers, L. E. M.; Vankelecom, I. F. J.; Jacobs, P. A.; Vos, D. E. D.; Catal. Lett. 2005, 102, 57.

11. Choudhary, T. V.; Goodman, D. W.; Appl. Catal., A 2005, 291, 32.

12. Sun, T.; Chan, M. L. H.; Quek, C. H.; Yu, H.; J. Biotechnol. 2004, 111, 169.

13. Wang, H.; Liu, Y.; Yang, Y.; Deng, T.; Shen, G.; Yu, R.; Anal. Biochem. 2004, 324, 219.

14. Wang, H.; Wang, C.; Lei, C.; Wu, Z.; Shen, G.; Yu, R.; Anal. Bioanal. Chem. 2003, 377, 632.

15. Krpetic, Z.; Porta, F.; Scarì, G.; Gold Bulletin 2006, 39, 66.

16. Liu, F. K.; Hsieh, S. Y.; Ko, F. H.; Chu, T. C.; Colloids Surf., A 2003, 231, 31.

17. Zhang, P.; Zhang, S.; Li, J.; Liu, D.; Guo, Z. X.; Ye, C.; Zhu, D.; Chem. Phys. Lett. 2003, 382, 599.

18. Wang, L.; Bai, J.; Huang, P.; Wang, H.; Zhang, L.; Zhao, Y.; Electrochem. Commun. 2006, 8, 1035.

19. Zhao, W.; Xu, J. J.; Shi, C. G.; Chen, H. Y.; Electrochem. Commun. 2006, 8,773 .

20. Zhuo, Y.; Yuan, R.; Chai, Y.; Zhang, Y.; Li, X.; Wang, N.; Zhu, Q.; Sens. Actuators, B 2006, 114, 631.

21. Raj, C. R.; Okajima, T.; Ohsaka, T.; J. Electroanal. Chem. 2003, 543, 127.

22. Zhang, L.; Ruo, R.; Chai, Y.; Chen, S.; Wang, N.; Zhu, Q.; Biochem. Eng. J. 2006, 28, 231.

23. Zhang, L.; Yuan, R.; Huang, X.; Chai, Y.; Cao, S.; Electrochem. Commun. 2004, 6, 1222 .

24. Yuan, R.; Zhang, L.; Li, Q.; Chai, Y.; Cao, S.; Anal. Chim. Acta 2005, 531, 1.

25. Zhuo, Y.; Yuan, R.; Chai, Y.; Zhang, Y.; Li, X. L.; Zhu, Q.; Wang, N.; Anal. Chim. Acta 2005, 548, 205.

26. Tang, D.; Yuan, R.; Chai, Y.; Fu, Y.; Electrochem. Commun. 2005, 7, 177.

27. Qu, S.; Li, H.; Peng, T.; Gao, Y.; Qiu, J.; Zhu, C.; Mater. Lett. 2004, 58 , 1427.

28. Yang, Y.; Matsubara, S.; Nogami, M.; Shi, J.; Huang, W.; Nanotechnology 2006, 17, 2821.

29. Lei, C. X.; Hu, S. Q.; Gao, N.; Shen, G. L.; Yu, R. Q.; Bioelectrochemistry 2004, 65, 33 .

30. Ghosh, A.; Patra, C. R.; Mukherjee, P.; Sastry, M.; Kumar, R.; Microporous Mesoporous Mater. 2003, 58, 201.

31. Lu, G.; Ji, D.; Qian, G.; Qi, Y.; Wang, X.; Suo, J.; Appl. Catal., A 2005, $280,175$.

32. Mafune, F.; Chem. Phys. Lett. 2004, 397, 133.

33. Hayashi, Y.; Takizawa, H.; Inoue, M.; Niihara, K.; Suganuma, K.; Transactions on Electronics Packaging Manufacturing 2005, 28, 338.

34. Stoleru, V. G.; Towe, E.; Microelectron. Eng. 2005, 81, 358.

35. Pereira, F. C.; Bergamo, E. P.; Zanoni, M. V. B.; Moretto, L. M.; Ugo, P.; Quim. Nova 2006, 29, 1054.

36. Martin, C. R.; Science 1994, 266, 1961.

37. Menon, V. P.; Martin, C. R.; Anal. Chem. 1995, 67, 1920.

38. Hulteen, J. C.; Martin, C. R.; J. Mater. Chem. 1997, 7, 1075.

39. Martin, C. R.; Chem. Mater. 1996, 8, 1739.

40. Foss, C. A. J.; Hornyak, G. L.; Stockert, J. A.; Martin, C. R.; J. Phys. Chem. 1994, 98, 2963.

41. Sun, Y.; Xia, Y.; Anal. Chem. 2002, 74, 5297.

42. Stranik, O.; McEvoy, H. M.; McDonagh, C.; MacCraith, B. D.; Sens. Actuators, B 2005, 107, 148.

43. Daniel M. C.; Astruc, D.; Chem. Rev. 2004, 104, 293.

44. Foss, C. A. J.; Hornyak, G. L.; Stockert, J. A.; Martin, C. R.; J. Phys. Chem. 1992, 96, 7497.

45. Schonenberger, C.; Zande, B. M. I.; Fokkink, L. G. J.; Henny, M.; Schmid, C.; Kruger, M.; Huber, R.; Birk, H.; Staufer, U.; J. Phys. Chem. B 1997, $101,5497$. 